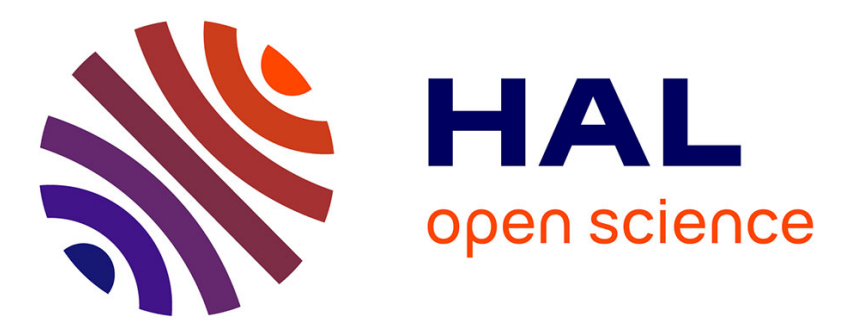

\title{
Relationships between nonlinear and space-variant linear models in hyperspectral image unmixing
}

\author{
Lucas Drumetz, Bahram Ehsandoust, Jocelyn Chanussot, Bertrand Rivet, \\ Massoud Babaie-Zadeh, Christian Jutten
}

\section{- To cite this version:}

Lucas Drumetz, Bahram Ehsandoust, Jocelyn Chanussot, Bertrand Rivet, Massoud Babaie-Zadeh, et al. Relationships between nonlinear and space-variant linear models in hyperspectral image unmixing. IEEE Signal Processing Letters, 2017, 24 (10), pp.1567-1571. 10.1109/LSP.2017.2747478 . hal01581520

\section{HAL Id: hal-01581520 \\ https://hal.science/hal-01581520}

Submitted on 4 Sep 2017

HAL is a multi-disciplinary open access archive for the deposit and dissemination of scientific research documents, whether they are published or not. The documents may come from teaching and research institutions in France or abroad, or from public or private research centers.
L'archive ouverte pluridisciplinaire HAL, est destinée au dépôt et à la diffusion de documents scientifiques de niveau recherche, publiés ou non, émanant des établissements d'enseignement et de recherche français ou étrangers, des laboratoires publics ou privés. 


\title{
Relationships between nonlinear and space-variant linear models in hyperspectral image unmixing
}

\author{
Lucas Drumetz, Member, IEEE, Bahram Ehsandoust, Jocelyn Chanussot, Fellow, IEEE, \\ Bertrand Rivet, Massoud Babaie-Zadeh, Senior Member, IEEE and Christian Jutten, Fellow, IEEE
}

\begin{abstract}
Hyperspectral image unmixing is a source separation problem whose goal is to identify the signatures of the materials present in the imaged scene (called endmembers), and to estimate their proportions (called abundances) in each pixel. Usually, the contributions of each material are assumed to be perfectly represented by a single spectral signature and to add up in a linear way. However, the main two limitations of this model have been identified as nonlinear mixing phenomena and spectral variability, i.e. the intraclass variability of the materials. The former limitation has been addressed by designing non linear mixture models, while the second can be dealt with by using (usually linear) space varying models. The typical example is a linear mixing model where the sources can vary from one pixel to the other. In this letter, we show that a recent variability model can also estimate the abundances of nonlinear mixtures to some extent. We make the theoretical connection between nonlinear models and this variability model, and confirm it with experiments on nonlinearly generated synthetic datasets.
\end{abstract}

Index Terms-Hyperspectral imaging, remote sensing, spectral unmixing, nonlinear mixtures, endmember variability

\section{INTRODUCTION}

$\mathbf{H}$ YPERSPECTRAL imaging is a nonconventional imaging technique which acquires information in many narrow and contiguous wavelengths of the electromagnetic spectrum, usually in the visible and near infrared domains. Every pixel of the resulting multivariate images is a complete reflectance spectrum. This fine spectral resolution allows an accurate identification of the materials present in the observed scene [1].

However, the spatial resolution of such images is more limited than conventional color or gray level images. As a result, several materials of interest are often present in the field of view of a given pixel. The observed spectrum is then a mixture of the contributions of each material. The inverse problem which consists in finding, for a new image, the signatures of the materials of the scene, and to estimate their proportions in each pixel is called spectral unmixing [2], [3]. This problem can be seen as a blind source separation problem.

Usually, a linear mixing model (LMM) is assumed to model the relationship between the observed data, the spectra of the pure materials (called endmembers), and the proportions (called abundances). The hyperspectral image is represented

L. Drumetz, B. Ehsandoust, J. Chanussot, B. Rivet and C. Jutten are with GIPSA-lab, CNRS, Grenoble Alpes University, F-38402 Saint Martin d'Heres Cedex, France (e-mail: \{lucas.drumetz;bahram.ehsandoust;jocelyn.chanussot; bertrand.rivet;christian.jutten\} @ gipsa-lab.grenoble-inp.fr).

L. Drumetz and J. Chanussot are also with the Department of Mathematics at the University of California, Los Angeles, 90095 Los Angeles, CA, USA

B. Ehsandoust and M. Babaie-Zadeh are also with Sharif University of Technology, Electrical Engineering Department, Tehran, Iran (e-mail: bahram.ehsandoust@gipsa-lab.grenoble-inp.fr, mbzadeh@yahoo.com).

This work has been supported by the 2012 ERC Advanced Grant project CHESS (Grant \# 320684), as well as the Agence Nationale de la Recherche and the Direction Générale de 1'Armement, project ANR-DGA APHYPIS, under grant ANR-16 ASTR-0027-01. L. Drumetz was also supported by a Campus France outgoing postdoctoral mobility grant, PRESTIGE-2016-4 0006. as a matrix $\mathbf{X} \in \mathbb{R}^{L \times N}$, where $L$ is the number of considered wavelengths, and $N$ is the number of pixels in the image. The endmembers are gathered in the columns of a matrix $\mathbf{S} \in \mathbb{R}^{L \times P}$, where $P$ is the number of considered materials. The abundance coefficients for each pixel and each material are stored in a matrix $\mathbf{A} \in \mathbb{R}^{P \times N}$. Then, for a given pixel $n$, the observed spectrum $\mathbf{x}_{n} \underset{P}{\in} \mathbb{R}^{L}$, the LMM writes:

$$
\mathbf{x}_{n}=\sum_{p=1}^{P} a_{p n} \mathbf{s}_{p}+\mathbf{e}_{n}
$$

where $\mathbf{e}_{n}$ is an additive noise, often assumed to be zero mean Gaussian-distributed, with an isotropic covariance matrix. The endmembers, being reflectance spectra, are constrained to be nonnegative. In addition, the abundances are proportions, so they are usually constrained to be positive, and to sum to one in each pixel. Geometrically, the LMM constrains the data to live in a simplex spanned by the endmembers. In many cases, the LMM is a reasonable approximation of the physics of the mixtures. However, in more complex cases nonlinear mixture models are necessary, for instance when rays of light undergo multiple reflections before reaching the sensor (e.g. in tree canopies) [4], [5].

This issue fostered research on nonlinear mixing models and the corresponding unmixing algorithms (e.g. [6]-[8]). A popular choice is the class of linear-quadratic models, which takes into account second order interactions between materials, under the form of product spectra $\mathbf{s}_{p} \odot \mathbf{s}_{q}$, where $\odot$ is the Hadamard (elementwise) product:

$$
\mathbf{x}_{n}=\sum_{p=1}^{P} a_{p n} \mathbf{s}_{p}+\sum_{p=1}^{P} \sum_{q=p}^{P} b_{p q n} \mathbf{s}_{p} \odot \mathbf{s}_{q}+\mathbf{e}_{n}
$$

where $b_{p q n}$ are positive quadratic interaction coefficients for each pixel $n$ and each pair of materials $(p, q)$. The higher order interactions are usually omitted, since they are considered to have a low contribution to the final at-sensor reflectance. The data is now bound to lie in a nonlinear manifold which is more complex than a simplex. A similar, but more restrictive model is given by the Generalized Bilinear Model (GBM) [9], which assumes that the coefficient of a nonlinear interaction term is proportional to the abundances of the materials involved:

$$
\mathbf{x}_{n}=\sum_{p=1}^{P} a_{p n} \mathbf{s}_{p}+\sum_{p=1}^{P} \sum_{q=p}^{P} \gamma_{p q n} a_{p n} a_{q n} \mathbf{s}_{p} \odot \mathbf{s}_{q}+\mathbf{e}_{n}
$$

where the importance of a nonlinear term is now governed by the abundances and parameter $\gamma_{p q n}$.

The other limitation comes from the representation of a single endmember by a unique spectral signature. This is a very convenient approximation, but an endmember is actually more accurately described by a collection of signatures, which account for the intra-class variability of that material [10] Each pixel can now be explained by different variants of the 
materials. Many physical phenomena can induce variations on the spectra of pure materials, be it a change in their physico-chemical composition, or the topography of the scene, which locally changes the incidence angle of the light and the viewing angle of the sensor. This phenomenon is referred to as endmember variability [11]-[13]. A physics-inspired model to explain illumination induced variability is the Extended Linear Mixing Model (ELMM) [14], which writes:

$$
\mathbf{x}_{n}=\sum_{p=1}^{P} a_{p n} \psi_{p n} \mathbf{s}_{p}+\mathbf{e}_{n}
$$

where $\psi_{p n}$ is a positive scaling factor whose effect is to rescale locally each endmember, the variations between variants of the same material due to changing illumination conditions being reasonably well explained by a scaling variation. Geometrically, the data may now lie inside a convex cone spanned by the endmembers. More specifically, each pixel belongs to a simplex, whose vertices can slide on lines (passing through the origin) which correspond to the edges of the convex cone.

Spectral variability and nonlinear mixtures are physically very different phenomena. Mathematically, spectral variability essentially amounts to using a space varying (usually linear) mixing model, while a general nonlinear mixing model is spatially invariant. Both phenomena have been considered simultaneously in recent works, e.g. by incorporating scaling factors in a bilinear mixing model [15], or by considering a residual based-model for the deviations from the LMM [16]. In [17], the joint consideration of both nonlinearities (through a linear-quadratic model) and spectral variability was experimentally shown not to give substantially better abundance estimation results than considering endmember variability alone. Since the dataset used was acquired over a urban area, where both phenomena were expected to be nonnegligible, results of [17] suggest that using a nonlinear model along with a variability model was not necessary, and that the latter can already handle nonlinear effects to some extent.

In this letter, following the ideas of [18], we provide theoretical insight to these results, by showing that there is a mathematical connection between both approaches. We show that a local Taylor expansion of a generic nonlinear model can be related to a variant of the spatially varying ELMM. This derivation, as well as the experiments, show that the ELMM has the ability to recover abundances from nonlinear mixtures, even though it was derived from physical considerations about endmember variability in linear mixtures.

The remainder of this letter is organized as follows: Section II shows the mathematical relationships between a general nonlinear model and the ELMM. Then, section III presents some results on synthetic datasets to experimentally confirm the theoretical derivation. Concluding remarks are finally gathered in section IV.

\section{CONNECTION BETWEEN NONLINEAR MODELS AND} VARIABILITY MODELS

A generic (noise free) nonlinear mixing model can be expressed, for a given pixel $n$ and wavelength $l$, as:

$$
x_{l n}=f_{n}\left(s_{l 1}, s_{l 2}, \ldots, s_{l P}\right)
$$

where $s_{l p}$ is the value of endmember $p$ at wavelength $l$, and $f_{n}: \mathbb{R}^{P} \rightarrow \mathbb{R}$ is a generic nonlinear function, which does not depend on the considered spectral band. Assuming the nonlinear function $f_{n}$ is sufficiently smooth, and that the sources are allowed to vary, we can perform an $M^{\text {th }}$ order Taylor expansion in $(0,0, \ldots, 0)$ :

$$
\begin{aligned}
& x_{l n}= f_{n}(\mathbf{0})+\mathbf{s}_{l:}^{\top} \nabla f_{n}(\mathbf{0})+\mathbf{s}_{l:}^{\top} \nabla^{2} f_{n}(\mathbf{0}) \mathbf{s}_{l:} \\
&+\cdots+o\left(\left\|\mathbf{s}_{l:}\right\|^{M}\right) \\
&=\sum_{p=1}^{P} \frac{\partial f_{n}}{\partial s_{l p}}(\mathbf{0}) s_{l p}+\sum_{p=1}^{P} \sum_{q=1}^{P} \frac{\partial^{2} f_{n}}{\partial s_{l p} \partial s_{l q}}(\mathbf{0}) s_{l p} s_{l q} \\
&+\cdots+o\left(\left\|\mathbf{s}_{l:}\right\|^{M}\right)
\end{aligned}
$$

where we have discarded the constant term (i.e. we assume that $\left.f_{n}(\mathbf{0})=0\right)$, and where $\mathbf{s}_{l:}=\left[s_{l 1}, \ldots, s_{l P}\right]^{\top} \in \mathbb{R}^{P}$. Note that even though this expansion is performed in $\mathbf{0}$, the error term $o\left(\left\|\mathbf{s}_{l:}\right\|^{M}\right)$ is likely to be small, because linear-quadratic and multilinear mixing models approximate the physics of hyperspectral imaging well. If the underlying nonlinear function is close to polynomial, we expect the coefficients of the expansion to be very close to the actual coefficients of the polynomial. In addition, even with a more general model, the expansion will also be valid in the neighborhood of $\mathbf{s}_{l}$ : with a high enough order $M$ of the expansion.

We change the notation of the coefficients of the expansion, keeping in mind their dependence with respect to the different variables of the model, and also change the indexing such that the identical second order terms are gathered in only one term:

$$
x_{l n}=\sum_{p=1}^{P} \alpha_{p n} s_{l p}+\sum_{p=1}^{P} \sum_{q=p}^{P} \beta_{p q n} s_{l p} s_{l q}+\cdots+o\left(\left\|\mathbf{s}_{l:}\right\|^{M}\right) \text {. }
$$

There is no dependence of the coefficients on the spectral band since we assumed the nonlinearity affects all spectral bands equally. If, following the physics of the problem, we assume the true nonlinear model is close to a multilinear model, that is a generalization of model (2) to higher order interaction terms, then we can safely assume that $\alpha_{p n} \approx a_{p n}$ and $\beta_{p q n} \approx b_{p q n}$, and then model (2) is a truncation at the second order of

$$
x_{l n}=\sum_{p=1}^{P} a_{p n} s_{l p}+\sum_{p=1}^{P} \sum_{q=p}^{P} b_{p q n} s_{l p} s_{l q}+\cdots+o\left(\left\|\mathbf{s}_{l:}\right\|^{M}\right) \text {. }
$$

On the other hand, if we factorize coefficient $\alpha_{p n} s_{l p}$ in the terms of Eq. (8), we obtain

$$
x_{l n}=\sum_{p=1}^{P} \alpha_{p n}\left(1+\sum_{q=p}^{P} \frac{\beta_{p q n}}{\alpha_{p n}} s_{l q}+\cdots+o\left(\left\|\mathbf{s}_{l:}\right\|^{M}\right)\right) s_{l p} .
$$

In order to make this factorization possible, we had to assume that all materials have a nonzero linear coefficient in pixel $n$. If the true model is multilinear, then these coefficients correspond to the abundances, and we simply have to remove the endmembers with zero abundance in pixel $n$ from the equation. By denoting the factor between the parentheses by $\psi_{l p n}$, and again by assuming the true model is close to multilinear, the first order coefficients are close to the abundances. Then, by factoring this coefficient and the endmember term $s_{l p}$, the rest of the expansion can be seen a scalar factor which depends on the pixel, band and material considered:

$$
x_{l n}=\sum_{p=1}^{P} a_{p n} \psi_{l p n} s_{l p}
$$


which is formally close to the variability model (4), with the notable exception that the scaling factor $\psi_{l p n}$ now depends on the wavelength. The ELMM is essentially a linear model where each endmember is allowed to vary spatially according to the law $\mathbf{s}_{p n}=\psi_{p n} \mathbf{s}_{p}$, where $\mathbf{s}_{p}$ is a reference signature for material $p$. The scaling factor $\psi_{p n}$ does not depend on the wavelength here. Note that model (11) is very general and may be too flexible to provide reliable performance without additional well chosen regularizations. Still, this shows that the space invariant (in terms of the endmembers) nonlinear model (5) can be locally approximated by a spatially varying linear model.

Finally, note that model (11) is more general than truncating model (9) at the second order, since the scaling factor incorporates information about the linear and quadratic terms of the expansion, but also about higher order terms.

\section{EXPERIMENTAL RESULTS}

In this section, we present experimental evidence of the fact that in certain situations, the ELMM can indeed estimate the abundances when the mixing model is nonlinear.

\section{A. Experimental setup}

We generated six different nonlinear synthetic datasets to test the three different models and different experimental conditions. First, we randomly selected three endmembers with 224 spectral bands from the USGS spectral library [19]. The abundances were generated using $200 \times 200$ Gaussian random fields and comply with the positivity and sum-to-one constraints. The endmembers and abundances used (shown in the top row of Fig. 1) are the same for all the tested models. We considered two levels of nonlinearity (moderate and high, depending on the magnitude of the coefficients) for each model.

All the resulting hyperspectral images are then of size $200 \times$ $200 \times 224$. We used the three following models to generate the datasets: the linear-quadratic Generalized Bilinear Model (GBM) (3), a a third order (trilinear) model, which extends model (3) to third order interactions, and the Multi-Linear Mixing (MLM) model of [20].

For the GBM, for each material, the nonlinear interaction coefficients $\gamma_{p q n}$ were generated using mixtures of Gaussians (so as to give them a spatial coherence), and are positive.

For the third order model, we used the same second order coefficients as in the previous case. The third order interaction coefficients were generated using mixtures of Gaussians.

The MLM considers interactions of possibly any order, but was derived from very different considerations than the linearquadratic or third order models. For instance, the derivation of this model leads to higher order interactions which result in a decrease of the total reflectance, rather than in the addition of a positive term to the linear model. This dataset will be used to test the performance in situations where the expansion (8) may be a worse approximation of the data than with a purely polynomial model. A pixel is generated using the following equation:

$$
\mathbf{x}_{n}=\frac{\left(1-P_{n}\right) \mathbf{S} \mathbf{a}_{n}}{1-P_{n} \mathbf{S} \mathbf{a}_{n}}+\mathbf{e}_{n}
$$

where $P_{n}$, if positive, represents the probability that, within the field of view of pixel $\mathbf{x}_{n}$, any ray of light (after any number of nonlinear interactions) undergoes an additional nonlinear interaction. In this case, we expect the polynomial model-based algorithms to provide poor results, because the constraints on the parameters cannot model decreases in total reflectance, but only increased reflectance w.r.t. the linear model. This case is possible in the MLM, by considering negative values for $P_{n}$ (see [20] for possible physical explanations). Values for $P_{n}$ were generated using mixtures of Gaussians, with values in the range $[-0.5,0]$ for the low nonlinearity level ([-0.75,0] for high nonlinearity level) for negative $P_{n}$, and in the range $[0,0.5]$ $\left([0,0.75]\right.$ for the high nonlinearity level) for positive $P_{n}$. In all cases, the noise was assumed to be Gaussian distributed with an isotropic covariance matrix, such that the signal to noise ratio is $30 \mathrm{~dB}$. Values are in the range $[0,0.5]$ for the low nonlinearity level $([0,0.75]$ for the high nonlinearity level) for all nonlinear coefficients.

We run and compare four different unmixing algorithms to estimate the abundances (assuming the endmember matrix $\mathbf{S}$ is known beforehand).

The Fully Constrained Least Squares Unmixing (FCLSU) algorithm of [21] is a least squares estimation of the abundances, with the abundance nonnegativity and sum-to-one constraints:

$$
\underset{\mathbf{A} \in \Delta_{P}}{\arg \min } \frac{1}{2}\|\mathbf{X}-\mathbf{S A}\|_{F}^{2}
$$

where $\mathbf{A} \in \Delta_{P}$ means that each column of $\mathbf{A}$ belongs to the unit simplex with $P$ vertices, and $\|\cdot\|_{F}$ is the Frobenius norm.

We also use a Linear-Quadratic unmixing strategy, very close to the one used in [22]. We store all the second order interaction spectra $\mathbf{s}_{p} \odot \mathbf{s}_{q}$ in a matrix $\mathbf{M} \in \mathbb{R}^{L \times P(P+1) / 2}$. Then model (2) can be rewritten in a matrix form, and we can estimate the abundances and nonlinear coefficients with the following optimization problem:

$$
\underset{\mathbf{A} \in \Delta_{P}, \mathbf{B} \geq \mathbf{0}}{\arg \min } \frac{1}{2}\|\mathbf{X}-\mathbf{S A}-\mathbf{M B}\|_{F}^{2}
$$

where $\mathbf{B} \in \mathbb{R}^{P(P+1) / 2 \times N}$ gathers all the nonlinear interaction coefficients, for all possible pairs of materials and all pixels. This problem is convex with respect to $\{\mathbf{A}, \mathbf{B}\}$ taken simultaneously, and separable with respect to those two variables (the constraints on each block are different, however), so we can obtain the global minimum by using an iterative procedure: we alternate a minimization of the function w.r.t. A, keeping $\mathbf{B}$ fixed and vice versa. Each minimization amounts to solving a either a nonnegative or fully constrained least squares problem. This model does not exactly correspond to (3), because here the nonlinear coefficients do not depend on the abundances.

We also adapt the previous algorithm to the third order case. By simply augmenting matrix $\mathbf{B}$ to include third order endmembers, we can handle the third order case using the same algorithm.

The ELMM unmixing algorithm, which, in its simplest form [23], solves the following optimization problem (nonconvex, but convex ${ }_{N}$ w.r.t. each block of variables):

$$
\underset{\mathbf{A} \in \Delta_{P}, \mathcal{S}, \boldsymbol{\psi}}{\arg \min } \frac{1}{2} \sum_{n=1}^{N^{2}}\left(\left\|\mathbf{x}_{n}-\mathbf{S}_{n} \mathbf{a}_{n}\right\|_{2}^{2}+\lambda_{S}\left\|\mathbf{S}_{n}-\mathbf{S} \boldsymbol{\psi}_{n}\right\|_{F}^{2}\right)
$$

where $\mathcal{S} \in \mathbb{R}^{L \times P \times N}$ gathers all the endmember signatures, for all pixels and all materials, $\mathbf{S}_{n} \in \mathbb{R}^{L \times P}$ is a slice of $\mathcal{S}$ corresponding to the local endmember matrix for pixel $n$, and $\psi_{n} \in \mathbb{R}^{P \times P}$ is a diagonal matrix whose diagonal elements are the scaling factors corresponding to pixel $n$, for all the materials. 


\begin{tabular}{|c|c|c|c|c|c|c|c|c|}
\hline Algorithm Model & \multicolumn{2}{|c|}{ GBM } & \multicolumn{2}{|c|}{ Third order model } & \multicolumn{2}{|c|}{$\operatorname{MLM}\left(P_{n} \leq 0\right)$} & \multicolumn{2}{|c|}{$\operatorname{MLM}\left(P_{n} \geq 0\right)$} \\
\hline FCLSU & 0.2329 & 0.3 & 0.3136 & 0 & 0.1686 & 0.2271 & 0.1939 & 0.2730 \\
\hline Linear-Quadratic & 0.031 & $\overline{0.02}$ & 0.0 & 0.1 & 0.1 & 0.1 & 0.1 & 0.2730 \\
\hline Third & 0.03 & & & & & & 0.1941 & \\
\hline ELMM & 0.0395 & 0.0562 & 0.0583 & 0.0874 & 0.1001 & 0.1400 & 0.1107 & 0.1680 \\
\hline
\end{tabular}

\section{TABLE I}

$R M S E(\hat{\mathbf{A}})$ VALUES FOR ALL CONFIGURATIONS. THE BEST RESULT FOR EACH CASE IS IN BOLD. THE LEFT (RESP. RIGHT) SIDE OF EACH CELL

CORRESPONDS TO A MODERATE (RESP. HIGH) LEVEL OF NONLINEARITY.

$\lambda_{S}$ is a regularization parameter forcing the local endmembers to be more or less close to scaled versions of the references. The optimization is performed by iterating minimization steps w.r.t. each of the three blocks of variables.

We initialize the last three algorithms with the results of the LMM, and stop them whenever the relative variation (in Frobenius norms) of the abundance matrix goes below $\epsilon_{\mathbf{A}}=10^{-3}$. The values of parameter $\lambda_{S}$ for the ELMM were empirically set (to obtain the best performance) to 1.5 (resp. 5) for the moderately (resp. highly) nonlinear GBM dataset, to 7 (resp. 6) for the moderately (resp. highly) nonlinear third order model, to 1 for the negative $P_{n}$ MLM datasets, and to 0.5 for the positive $P_{n}$ MLM data.

\section{B. Results}

For each dataset and algorithm, we computed the overall abundance Root Mean Squared Errors (RMSE between the true abundances $\mathbf{a}_{n}$ and the estimated ones $\hat{\mathbf{a}}_{n}$ ):

$$
\operatorname{RMSE}(\hat{\mathbf{A}})=\frac{1}{N \sqrt{L}} \sum_{n=1}^{N}\left\|\mathbf{a}_{n}-\hat{\mathbf{a}}_{n}\right\|_{2}
$$

These quantities, for all algorithms and datasets, are gathered in Table I. As expected, the LMM based algorithm (FCLSU) provides a poor abundance estimation, which gets worse and worse when nonlinearity or model complexity increases. Not surprisingly, the Linear-Quadratic based method obtains the best abundance estimation results on the GBM data, for both levels of nonlinearity. This is because both models are formally similar and just differ in that the coefficients of the GBM depend on the abundances. The ELMM obtains relatively good performance on this dataset as well. Indeed, the values of the scaling factors are all greater than 1 (whereas they are not explicitly constrained, and can be either lower or greater than 1 in endmember variability scenarios), which matches Eq. (10). The difference in performance may be explained by the fact that the ELMM considers scaling factors to be independent of the spectral band, which is not the case in Eq. (11).

When third order terms are included in the model, all unmixing algorithms achieve worse (but acceptable, except for FCLSU) performance than in the second order case. But more importantly, in that case, the ELMM obtains better results than the Linear-Quadratic or the third order algorithms. The reason for this is that, following the derivation of section II, the scaling factor of the ELMM is able to incorporate information corresponding to higher order terms, whereas using the polynomial algorithms means truncating the expansion to second or third-order terms. The third order algorithm may require additional regularizations, such as sparsity, to avoid overfitting the data, due to the large size of the endmember matrix.

We show in Fig. 1 the true and estimated abundances by the four tested algorithms with the same third order model data. The visual results confirm the quantitative ones on the fact that the LMM fails because of the nonlinearities. The polynomial

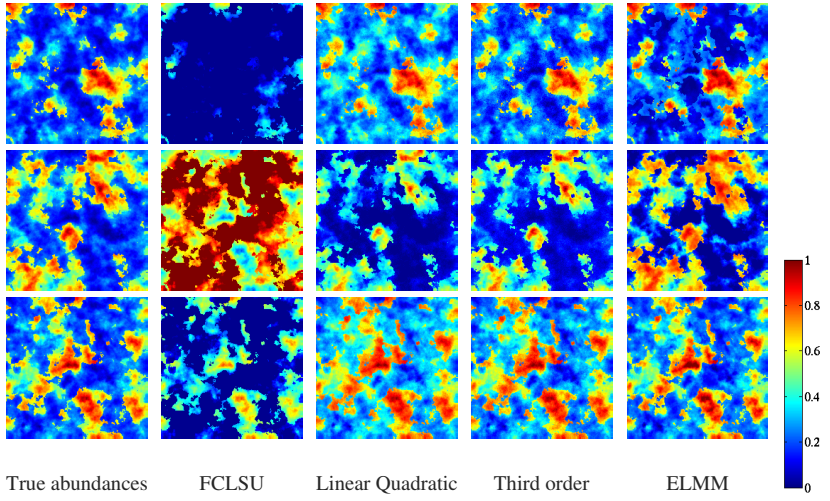

Fig. 1. True abundances (leftmost column) and estimated abundances by the four tested algorithms on the third order model data with a moderate level of nonlinearity (in the columns, from left to right: FCLSU, Linear-Quadratic algorithm, Third order algorithm, ELMM).

unmixing algorithms obtain better estimations, but far from perfect, especially for material two. Finally, the ELMM, even if it was not designed for this purpose, is able to obtain abundance maps which match best the true ones. There are still some discrepancies for material one, but the overall abundance estimation is close to the true abundance maps and visually less noisy than the polynomial ones.

For the case of the MLM data, all algorithms obtain relatively poor results. This is due to the fact that the MLM has a more general expression than a polynomial model and is then much less accurately approximated by the Taylor expansion. The closest abundances to the ground truth in that case are still those of the ELMM. This happens both for positive and negative values of $P_{n}$ : in the former case, the scaling factors are lower than 1 to account for the decrease in reflectance. The linear, second and third order models perform equally bad, because the nonlinear coefficients are all close to 0 , so as not to increase the total reflectance. In the negative case, the scaling factors are always greater than 1 because the total reflectance is increased w.r.t. the LMM.

\section{CONCLUSION}

In this letter, we showed that a general nonlinear mixture, approximated locally by a Taylor expansion, is formally very similar to the Extended Linear Mixing Model, in which scaling factors model the variability of the endmembers. The similarity only requires that the magnitude of the error in the expansion is not too large, e.g. if the true model is close to polynomial. This general theoretical result was experimentally validated for hyperspectral image unmixing by comparing the performance of 4 unmixing algorithms on 6 datasets generated by 3 different nonlinear models in different conditions. Experimental results show the efficacy and accuracy of ELMM algorithm for any of the tested nonlinear models, through a better abundance estimation performance than the competing algorithms. The ELMM is also proven to handle general nonlinear mixtures better than polynomial model-based algorithms.

However, these results raise the question of how to efficiently extract information related to both endmember variability and nonlinear mixtures at the same time. When nonlinear mixtures are involved at the same time as variability, separating the contributions of both phenomena might not be directly possible using the ELMM, even though abundance estimation is not impacted. 


\section{REFERENCES}

[1] J. Bioucas-Dias, A. Plaza, G. Camps-Valls, P. Scheunders, N. Nasrabadi, and J. Chanussot, "Hyperspectral remote sensing data analysis and future challenges," IEEE Geoscience and Remote Sensing Magazine, vol. 1, pp. 6-36, June 2013.

[2] J. Bioucas-Dias, A. Plaza, N. Dobigeon, M. Parente, Q. Du, P. Gader, and J. Chanussot, "Hyperspectral unmixing overview: Geometrical, statistical, and sparse regression-based approaches," IEEE Journal of Selected Topics in Applied Earth Observations and Remote Sensing, vol. 5, pp. 354-379, April 2012.

[3] W.-K. Ma, J. Bioucas-Dias, T.-H. Chan, N. Gillis, P. Gader, A. Plaza, A. Ambikapathi, and C.-Y. Chi, "A signal processing perspective on hyperspectral unmixing: Insights from remote sensing," IEEE Signal Processing Magazine, vol. 31, pp. 67-81, Jan 2014.

[4] R. Heylen, M. Parente, and P. Gader, "A review of nonlinear hyperspectral unmixing methods," IEEE Journal of Selected Topics in Applied Earth Observations and Remote Sensing, vol. 7, pp. 1844-1868, June 2014.

[5] N. Dobigeon, J. Y. Tourneret, C. Richard, J. C. M. Bermudez, S. McLaughlin, and A. O. Hero, "Nonlinear unmixing of hyperspectral images: Models and algorithms," IEEE Signal Processing Magazine, vol. 31, pp. 82-94, Jan 2014.

[6] I. Meganem, Y. Deville, S. Hosseini, P. Déliot, and X. Briottet, "Linearquadratic blind source separation using nmf to unmix urban hyperspectral images," IEEE Transactions on Signal Processing, vol. 62, pp. 18221833, April 2014.

[7] Y. Altmann, N. Dobigeon, and J. Y. Tourneret, "Unsupervised postnonlinear unmixing of hyperspectral images using a hamiltonian monte carlo algorithm," IEEE Transactions on Image Processing, vol. 23, pp. 2663-2675, June 2014.

[8] C. Févotte and N. Dobigeon, "Nonlinear hyperspectral unmixing with robust nonnegative matrix factorization," IEEE Transactions on Image Processing, vol. 24, pp. 4810-4819, Dec 2015.

[9] A. Halimi, Y. Altmann, N. Dobigeon, and J.-Y. Tourneret, "Nonlinear unmixing of hyperspectral images using a generalized bilinear model," IEEE Transactions on Geoscience and Remote Sensing, vol. 49, no. 11, pp. 4153-4162, 2011.

[10] A. Zare and K. Ho, "Endmember variability in hyperspectral analysis: Addressing spectral variability during spectral unmixing," IEEE Signal Processing Magazine, vol. 31, pp. 95-104, Jan 2014.

[11] P.-A. Thouvenin, N. Dobigeon, and J.-Y. Tourneret, "Hyperspectral unmixing with spectral variability using a perturbed linear mixing model," IEEE Transactions on Signal Processing, vol. 64, no. 2, pp. 525-538, 2016.

[12] A. Halimi, N. Dobigeon, and J. Y. Tourneret, "Unsupervised unmixing of hyperspectral images accounting for endmember variability," IEEE Transactions on Image Processing, vol. 24, pp. 4904-4917, Dec 2015.

[13] S. Henrot, J. Chanussot, and C. Jutten, "Dynamical spectral unmixing of multitemporal hyperspectral images," IEEE Transactions on Image Processing, vol. 25, pp. 3219-3232, July 2016.

[14] L. Drumetz, M. A. Veganzones, S. Henrot, R. Phlypo, J. Chanussot, and C. Jutten, "Blind hyperspectral unmixing using an extended linear mixing model to address spectral variability," IEEE Transactions on Image Processing, vol. 25, pp. 3890-3905, Aug 2016.

[15] A. Halimi, P. Honeine, and J. M. Bioucas-Dias, "Hyperspectral unmixing in presence of endmember variability, nonlinearity, or mismodeling effects," IEEE Transactions on Image Processing, vol. 25, no. 10, pp. $4565-4579,2016$.

[16] A. Halimi, J. M. Bioucas-Dias, N. Dobigeon, G. S. Buller, and S. McLaughlin, "Fast hyperspectral unmixing in presence of nonlinearity or mismodeling effects," IEEE Transactions on Computational Imaging, vol. 3, no. 2, pp. 146-159, 2017.

[17] C. Revel, Y. Deville, V. Achard, and X. Briottet, "A linear-quadratic unsupervised hyperspectral unmixing method dealing with intra-class variability," in IEEE Workshop on Hyperspectral Image and Signal Processing: Evolution in Remote Sensing (WHISPERS 2016), 2016.

[18] B. Ehsandoust, M. Babaie-Zadeh, and C. Jutten, "Blind source separation in nonlinear mixture for colored sources using signal derivatives," in International Conference on Latent Variable Analysis and Signal Separation, pp. 193-200, Springer, 2015.

[19] R. F. Kokaly, R. N. Clark, G. A. Swayze, K. E. Livo, T. M. Hoefen, N. C. Pearson, R. A. Wise, W. M. Benzel, H. A. Lowers, R. L. Driscoll, et al., "Usgs spectral library version 7," tech. rep., US Geological Survey, 2017.

[20] R. Heylen and P. Scheunders, "A multilinear mixing model for nonlinear spectral unmixing," IEEE Transactions on Geoscience and Remote Sensing, vol. 54, pp. 240-251, Jan 2016.
[21] D. Heinz and C.-I. Chang, "Fully constrained least squares linear spectral mixture analysis method for material quantification in hyperspectral imagery," IEEE Transactions on Geoscience and Remote Sensing, vol. 39, pp. 529-545, Mar 2001.

[22] N. Yokoya, J. Chanussot, and A. Iwasaki, "Nonlinear unmixing of hyperspectral data using semi-nonnegative matrix factorization," IEEE Transactions on Geoscience and Remote Sensing, vol. 52, no. 2, pp. 14301437, 2014.

[23] L. Drumetz, S. Henrot, M. A. Veganzones, J. Chanussot, and C. Jutten, "Blind hyperspectral unmixing using an extended linear mixing model to address spectral variability," in IEEE Workshop on Hyperspectral Image and Signal Processing: Evolution in Remote Sensing (WHISPERS 2015), 2015. 